\title{
固定化烟草核酮糖-1,5-二磷酸翔化酶/ 加氧酶的解离及重组作用
}

\author{
李立人 李粹芳 许仁邦
}

（中国科学院上海植物生理研究所）

\section{摘要}

本文将共价偶联于 CNBr 活化的 Sepharose 4B 上的烟草核酮糖-1，5-二磷 酸楸化酶/加氧酶用不同浓度尿素处理后,发现 $2-2.5 \mathrm{~mol} / \mathrm{L}$ 尿素可将小亚单位解离 下来而大亚单位仍偶联在载体上. $3 \mathrm{~mol} / \mathrm{L}$ 以上层素可将大亚单位 8 聚体， $\mathrm{L}_{3}$ 进一 步解离为单体. 因此, 酶是通过大亚单位上的 $\varepsilon$-氨基与载体相偶联的. 小亚单位的 解离量与酶活性的下降呈线性相关。将解离酶的层素浓度稀释至 $0.5 \mathrm{~mol} / \mathrm{L}$ ，解离的 小亚单位几乎全部结合到残缺小亚单位的固相酶上，酶的活性也接近全部恢复. 重 组小亚单位量与酶活性增加亦呈线性相关。结果表明：除大亚单位外，小亚单位对 维持酶的活性也有重要的作用。

核酮睢-1，5-二磷酸羧化酶/加氧酶 (简称 RuBP 羧化酶，EC 4.1.1.39) 是一个双功 能酶, 在植物光合二氧化碳固定和光呼吸碳氧化过程中起中心的作用. 高等植物的 RuBP 羧 化酶由 8 个大亚单位 ( $\mathrm{L}$, 分子量为 56kDa) 和 8 个小亚单位 ( $\mathrm{S}$, 分子量为 $14 \mathrm{kDa}$ ) 组 成. 现在已知酶的催化 ${ }^{[1]}$ 和活化 ${ }^{[2]}$ 的位置均在大亚单位上, 然而小亚单位的功能至今仍不清 楚. 解决这一问题最常用的方法是利用各种化学试剂解离 $\mathrm{L}_{8} \mathrm{~S}_{8}$ 酶的四级结构,然后研究其分

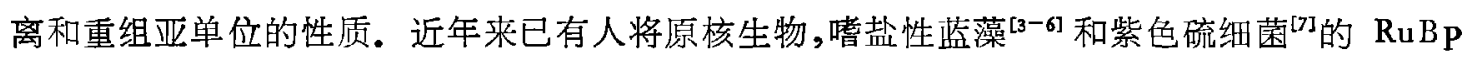
羧化酶进行解离和重组的研究. 对于高等植物, 仅早先 Nishimura 和 Akazawa ${ }^{[8]}$ 曾做过这 方面的工作,但他们的实验存在一些问题, 主要是重组酶活性的回收率太小䭪，未能达到有效 的解离和重组. 最近, Jodan 和 Chollet ${ }^{[7]}$ 重复了 Nishimura 和 Akazawa 的实验,并且用了 各种方法想克服聚合沉淀的问题,结果仍未能得到可察觉的重组活力. 因而，他们甚至声称， 高等植物来源的 RuBP 䍳化酶是不宜用来做这种解离和重组实验的.

我们将烟草 RuBP 羒化酶进行了固定化. 当固定化的酶进行解离后，在除去解离剂时， 由于载体的位阻，可避免亚单位之间的聚合沉淀作用. 因此，我们成功地进行了高等植物 $\mathrm{RuBP}$ 羧化酶的解离和重组的研究.

\section{一、材料和方法}

\section{1. 化学试剂}

核酮糖-1，5-二磷酸钠盐系 Sigma 产品, CNBr-Activated Sepharose 4B 系 Phamacia 
产品， $\mathrm{NaH}^{14} \mathrm{CO}_{3}$ 系中国医学科学院产品.

\section{2. 烟草 RuBP 䍴化酶结晶的制备}

取人工气候室栽培的烟草 (Nicotiana tobaccum) 革新一号按李立人等 ${ }^{[10]}$ 方法制备, 并重 结晶一次.

\section{3. 固定化 RuBP 羒化酶的制备}

基本上参考 $\mathrm{Chan}^{[11]}$ 的方法. 取适量重结晶的酶溶于偶联缓冲液 $\left(0.1 \mathrm{~mol}^{1 /} / \mathrm{L}\right.$ 磷陵缓冲液 $\mathrm{pH} 8.3 ， 0.5 \mathrm{~mol} / \mathrm{L} \mathrm{NaCl}$ ) 中. 将经溶胀并洗涤过的 $\mathrm{CNBr}$ 活化的 Sepharose $4 \mathrm{~B}$ 立即投入 含酶的偶联缓冲液中, 于 $25^{\circ} \mathrm{C}$ 振荡保温 $4 \mathrm{~h}$ 。抽滤并洗涤, 然后与 $0.2 \mathrm{~mol} / \mathrm{L}$ 甘氨酸 $(\mathrm{pH} 8.0)$ 混和,再于 $25^{\circ} \mathrm{C}$ 振荡保温 $2.5 \mathrm{~h}$. 抽滤并用含不同盐浓度和 $\mathrm{pH}$ 的 Tris 缓冲液交替洗涤. 将固


并在冰箱中保存. 固相酶的比活约为天然酶的 $30-40 \%$.

\section{4. 偶联蛋白含量的测定}

加人的酶蛋白量扣除在偶联过程中抽滤和洗下来的酶蛋白量（计算蛋白浓度 $\mathrm{mg} / \mathrm{ml}$ 为 消光系数 $\mathrm{O} . \mathrm{D}_{280 \mathrm{~nm}}^{\mathrm{Icm}}$ 乘以 0.7) 即为已偶联的酶蛋白量. 如用 $6 \mathrm{~N} \mathrm{HCl}$ 将固定化酶全部水解 然后进行氨基酸分析计算 CNBr-Sepharose $4 B$ 胶上的酶蛋白量, 其数值略低于上述扣除法. 本文对偶联之酶含量测定均按上述扣除法计算.

\section{RuBP 羚化酶活性的测定}

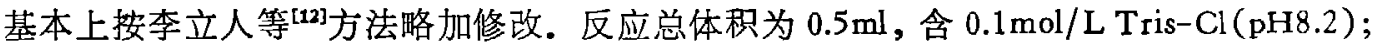
$10 \mathrm{mmol} / \mathrm{L} \mathrm{NaH}^{14} \mathrm{CO}_{3}(0.4 \mu \mathrm{ci} / \mu \mathrm{mol} / \mathrm{L}) ; 20 \mathrm{mmol} / \mathrm{LMgCl}_{2} ; 1 \mathrm{mmol} / \mathrm{L} \mathrm{DTT} ; 0.5 \mathrm{mmol} / \mathrm{L} \mathrm{RuBP}$ 和适量活化的天然或固相酶, 以加人 RuBP 开始反应。反应温度为 $30^{\circ} \mathrm{C}$, 反应时间为 $2 \mathrm{~min}$ (固定化酶反应时间为 $5 \mathrm{~min}$ ). 酶的活化是将结晶的酶溶解(固相酶则悬浮)于活化液，即 0.1 $\mathrm{mol} / \mathrm{LTris}-\mathrm{Cl} \mathrm{pH} 8.2$ (内含 $10 \mathrm{mmol} / \mathrm{L} \mathrm{NaHCO}_{3}, 20 \mathrm{mmol} / \mathrm{L} \mathrm{MgCl}_{2}, 0.1 \mathrm{~mol} / \mathrm{L} \mathrm{NaCl}$ )中, $50^{\circ} \mathrm{C}$ 保温 $10 \mathrm{~min}$. 固定化酶的转移以及在活化和反应时都在磁力搅拌下进行. 反应结束, 加入 0.2 $\mathrm{ml} 2 \mathrm{~N} \mathrm{HCl}$, 然后从中取出 $0.5 \mathrm{ml}$ 液体于内炼计数瓶中烘干. 冷却后加人 $0.5 \mathrm{ml}$ 水和 $4.5 \mathrm{ml}$ 内 炼液(闪歓液组份：6g PPO, 0.075g POPOP, 11 甲苯, 0.51 triton-x-100), 然后于 Beckman 1s 9800 液体闪淿计数器上计数.

\section{6. 凝胶柱层析}

取 $45 \mathrm{mg}$ 烟草 RuBP 羧化酶结晶悬浮液, 离心，沉淀溶解于 $2.5 \mathrm{ml}, 50 \mathrm{mmol} / \mathrm{L} \mathrm{tris}-$ $\mathrm{Cl}$ ( $\mathrm{pH} 8.0$ 内含 $0.2 \mathrm{mmol} / \mathrm{L} \mathrm{EDTA}, 5 \mathrm{mmol} / \mathrm{L}$ 巯基乙醇, $4.0 \mathrm{~mol} / \mathrm{L}$ 尿素) 之缓冲液中, $20^{\circ} \mathrm{C}$ 预保温约 $30 \mathrm{~min}$ 后用 Sephadex G-100 进行凝胶柱层析 (床体积约 $40 \mathrm{ml}$ ), 凝胶柱预先用以 上含尿素的缓冲液平衡, 用同样的缓冲液洗脱, 流速为 $20 \mathrm{ml} / \mathrm{h}$, 每 $1.2 \mathrm{ml}$ 分部蚊集, 并分别测 定其在 $280 \mathrm{~nm}$ 的光吸收值.

\section{7. 酶的解离作用}


$5 \mathrm{mmol} / \mathrm{L}$ 疏基乙醇)洗一次, 用上述 $\mathrm{tris}$ 缓冲液定容至 $1 \mathrm{ml}$, 然后加人高一倍的各种浓度之 尿素溶液（尿素溶解于上述 Tris 缓冲液中） $1 \mathrm{ml}$, 以达到所霖之各尿素浓度,于室温下振荡保 温 $10 \mathrm{~min}$, 并进行 SDS 凝胶电泳观察其谱带是小亚单位还是大亚单位. 固相部份用相应的不 同浓度尿素洗三次再用活化液洗三次,然后测定其酶活性. 


\section{SDS 凝胶电泳}

采用改良的 Weber ${ }^{[13]}$ 方法. 用考马斯亮蓝 R-250 染色.

\section{9. 酶的重组合作用}

取一定量的固相酶悬浮液,加入等体积的 $5 \mathrm{~mol} / \mathrm{L}$ 尿素(溶解于 $50 \mathrm{mmol} / \mathrm{L}$ Tris- $\mathrm{Cl} \mathrm{pH} 8.0$ ，

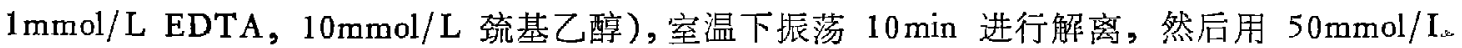
Tris- $\mathrm{Cl}$ ( $\mathrm{pH} 8.0$ 内含 $1 \mathrm{mmol} / \mathrm{L} \mathrm{EDTA}, 10 \mathrm{mmol} / \mathrm{L}$ 颈基乙醇)使尿素稀释至 $0.5 \mathrm{~mol} / \mathrm{L}$, 于 $25^{\circ} \mathrm{C}$ 振荡保温 $15 \mathrm{~min}$. 固相部分用活化液洗三次，然后测定酶活性. 不同小亚单位浓度的重组合 是在酶被尿素解离后,除去不同体积的上清液,然后再用 Tris- $\mathrm{Cl}$ 缓冲液如上稀释进行重组.

\section{二、结 果}

\section{1. 尿素对天然 RuBP 羧化酶的解离作用}

将烟草 $\mathrm{RuBP}$ 羒化酶结晶溶解于含尿素的 $\mathrm{Tris}-\mathrm{Cl}$ 缓冲液中进行预保温处理，然后用 Sephadex-G100 进行凝胶柱层析, 可观察到当尿素浓度达到 $4.0 \mathrm{~mol} / \mathrm{L}$ 时, RuBP 羒化酶明 显解离为二个峰（图 1). 将分部收集的二个高峰部份进行 SDS 凝胶电泳, 检测其分子量, 可看出分别是酶的大、小二个亚单位．如将收集到的二部份大、小亚单位分别进行透析以除 去其中的尿素，则可观察到大亚单位出现沉淀，而小亚单位仍保留在溶液中. 这说明大亚单 位在除去尿素后极易发生自身聚合形成沉淀，而小亚单位不会自身聚合，如将收集之大、小亚 单位混和在一起, 然后在琉基试剂存在下透析除去尿素, 也同样发生聚合沉淀. 上清液无羧化. 酶活力.

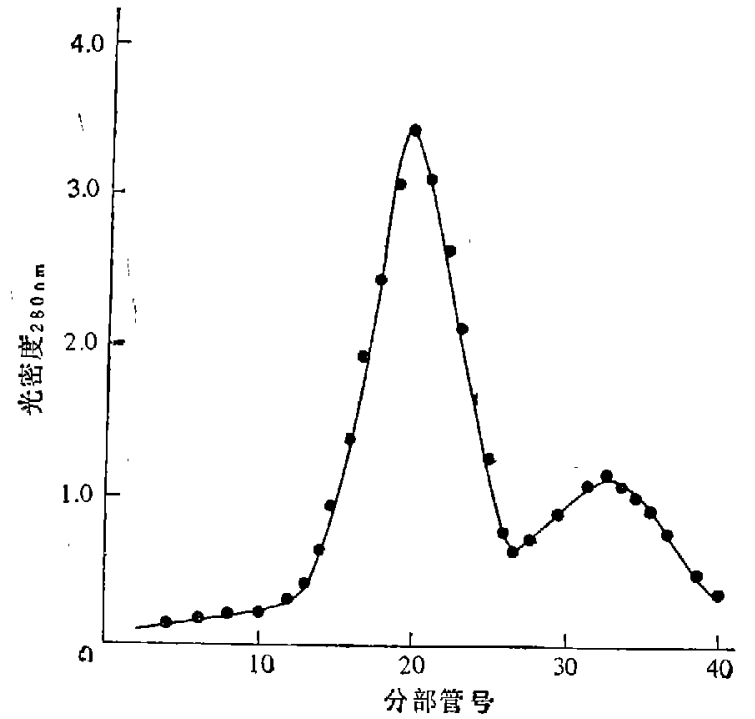

图 1 天然烟草 RuBP 羧化酶于 $4 \mathrm{~mol} / \mathrm{L}$ 尿素 处理后的凝胶柱层析

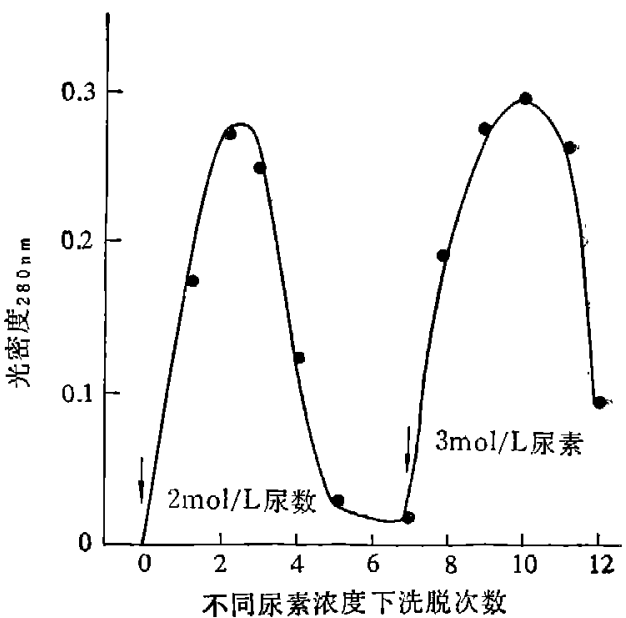

图 2 固定化 RuBP 羧化酶在 $2.0 \mathrm{~mol} / \mathrm{L}$ 及 $3.0 \mathrm{~mol} / \mathrm{L}$ 尿素浓度下解离的动力学过程

\section{2. 尿素对固定化 RuBP 羭化酶的解离作用}

将偶联于 $\mathrm{CNBr}$ 活化的 Sepharose $4 \mathrm{~B}$ 上的固定化烟草 $\mathrm{RuBP}$ 羧化酶用一定量 含 2.0 
$\mathrm{mol} / \mathrm{L}$ 尿素的 Tris 缓冲液洗脱多次，测定每次洗脱液的 $280 \mathrm{~nm}$ 的 O.D 值，并相对洗脱的 顺序作图 (图 2), 可观察到一个蛋白峰的出现. 然后, 用 $3.0 \mathrm{~mol} / \mathrm{L}$ 尿素也同样多次洗脱测定 则又出现一个蛋白峰.

将洗脱出现的二个蜂部份进行 SDS 凝胶电泳,则可观察到（图 3) 前一个蜂，即经 2.0 $\mathrm{mol} / \mathrm{L}$ 尿素解离出现的一条谱带是小亚单位. 后一个蜂,即经 $2.0 \mathrm{~mol} / \mathrm{L}$ 尿素处理并洗脱多次 后再经 3.0mol/L 尿素处理出现二条带;一条大亚单位带和一条弱的小亚单位带.

结果说明 $2.0 \mathrm{~mol} / \mathrm{L}$ 尿素使小亚单位解离下来,而大亚单位核， $\mathrm{L}_{3}$ 仍偶联在载体上，即 $\mathrm{L}_{8} \mathrm{~S}_{8} \rightarrow \mathrm{L}_{8}+8 \mathrm{~S}$. $3.0 \mathrm{~mol} / \mathrm{L}$ 尿素进一步使大亚单位核, $\mathrm{L}_{3}$ 解离为单体, 即 $\mathrm{L}_{8} \rightarrow 8 \mathrm{~L} .2 .0$ $\mathrm{mol} / \mathrm{L}$ 尿素仅使小亚单位解离下来, 而无大亚单位解离说明酶是通过部份大亚单位 的 $\varepsilon$-氨 基与 Sepharose 载体共价结合的.



图 3 固定化烟草 RuBP 羧化酶用 $2 \mathrm{~mol} / \mathrm{L}$ 和 $3 \mathrm{~mol} / \mathrm{L}$ 尿素依次洗脱后上清液的 SDS 凝胶电泳

(1- $2 \mathrm{~mol} / \mathrm{L}$ 尿索处理后的 SDS 电泳, $2-2 \mathrm{~mol} / \mathrm{L}$ 尿 素洗脱后再用 $3 \mathrm{~mol} / \mathrm{L}$ 㽷素处理的 SDS 电泳, 3-天 然酶的 SDS 电泳)



图 4 不同浓度尿素处理固定化烟草 RuBP 羧化酶后上清液的 SDS 凝胶电泳

$(1-1.0 \mathrm{~mol} / \mathrm{L}$ 尿素处理, $2-2.0 \mathrm{~mol} / \mathrm{L}$ 尿素外 理, $3-2.5 \mathrm{~mol} / \mathrm{L}$ 尿素处理, $4-3.0 \mathrm{~mol} / \mathrm{L}$ 尿 索处理, $5-4.0 \mathrm{~mol} / \mathrm{L}$ 尿素处理)

\section{3. 酶的解离程度与活性的关系}

用 $0-4 \mathrm{~mol} / \mathrm{L}$ 不同浓度的尿素溶液处理固定化的烟草 RuBP 羧化酶后, 取上清液进行 SDS 聚丙烯凝胶电泳,可发现（图 4) 小亚单位在 $2.0 \mathrm{~mol} / \mathrm{L}$ 尿素处理后开始解离,随尿素浓度 增加,小亚单位解离量增加. 当尿素浓度达 $3.0 \mathrm{~mol} / \mathrm{L}$ 时, 大亚单位明显解离.

从图 4 看出: 大亚单位的量总比小亚单位还要少, 这是由于部份大亚单位与 Sepharose 载体形成共价结合之故。

将 $0-4 \mathrm{~mol} / \mathrm{L}$ 不同浓度尿素处理后，离心并洗脱除去上清液后测定不同残缺程度的固定 化酶的活性. 结果表明 (图 5)：羒化酶活性的损先与亚单位的解离量成正比. 加氧酶活性的 变化也有相同的趋势(资料未列出). 图 5 活性曲线似乎说明存在有二个一级反应：第一个一 级反应酶活性下降速度较慢, 仅与小亚单位解离程度相关,第二个一级反应从 $2.0 \mathrm{~mol} / \mathrm{L}$ 尿素 浓度开始，酶活性下降速率较快，它与大、小亚单位的解离同时相关，这说明大、小亚单位分 别对酶的反应均有重要影响。 




图 5 不同浓度尿素下固定化 RuBP 羒化酶亚单位的解离程度与酶活性的关系

$$
(\cdot-\text { - 示活性, } \Delta-\Delta \text { 示光密度) }
$$

为了进一步说明小亚单位与酶活性的关系, 用 $2.0 \mathrm{~mol} / \mathrm{L}$ 尿素处理固定化的酶, 从不同尿 素处理时间可以看出（图 6(a)）：小亚单位的解离程度随处理时间的增加而增加，而酶活性则 随处理时间的增加而下降 (图 6(b)). 如以小亚单位的解离量相对酶活性作图, 可观察到小亚 单位解离的程度与酶活性成线性反比关系 (图 6(c)).

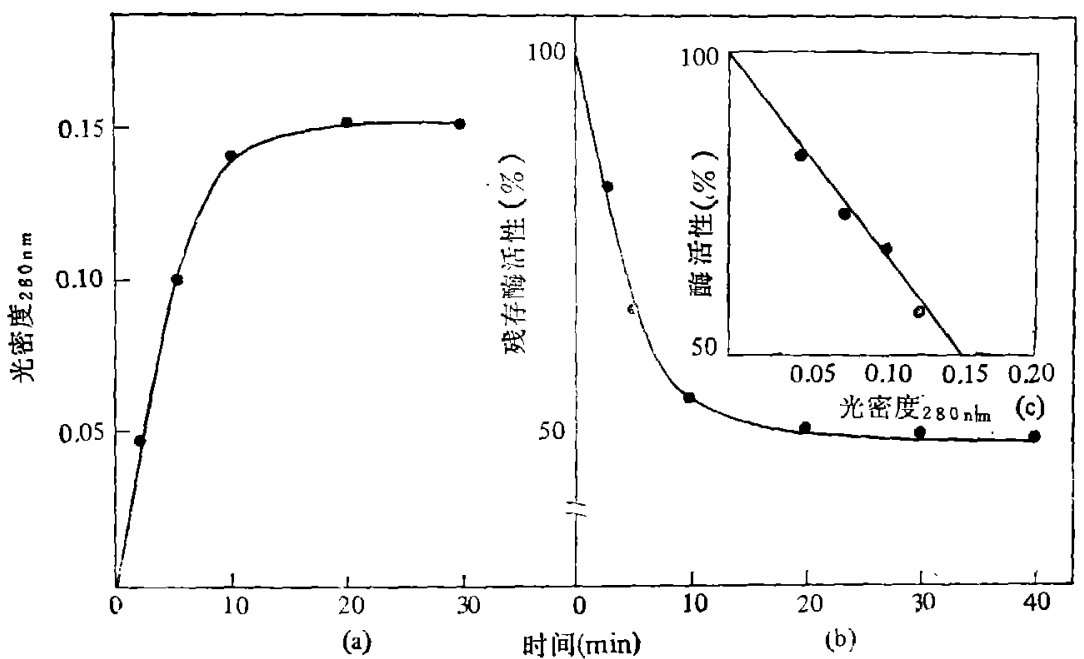

图 6 小亚单位解离量与固定化酶活性的动力学比较

\section{4. 亚单位的重组合作用}

用 $2.5 \mathrm{~mol} / \mathrm{L}$ 尿素解离固定化的烟草 RuBP 羧化酶后, 测定其上清液的 $280 \mathrm{~nm}$ 的光吸收 值,求出所解离之小亚单位量约为固定化酶小亚单位总量的 $50 \%$. 这种残缺小亚单位的固定化 
酶的活性约为全酶活性的 $50 \%$. 将 $2.5 \mathrm{~mol} / \mathrm{L}$ 尿素解离后的固定化酶悬浮液用含琉基乙醇的 Tris 缓冲液稀释 5 倍, 并在 $25^{\circ} \mathrm{C}$ 下振荡保温 $15 \mathrm{~min}$, 则解离下来的小亚单位又与残缺的固定化 酶发生重组合作用. 重组合的现象丰要表现在（表 1) 上清液的小亚单位含量降低到接近为 零, 而酶的活性上升到接近对照的水平, 重组合的酶活性几乎全部被回收. 如解离后分别除去 不同体积含小亚单位的上清液,然后再用 Tris 缓冲液稀释进行重组合,则可观察到 (图 7) 重 组合的小亚单位量与酶的活性成线性正相关.

表 1 固定化烟草 RuBP 羭化酶解离及重组后小亚单位含量与固相酶活性变化

\begin{tabular}{c|c|c|c}
\hline 处 & $\begin{array}{c}\text { 上 清 液 } \\
\text { 小亚单出含量 } \\
\text { (O. D. } 280 \mathrm{~nm})\end{array}$ & $\begin{array}{c}\text { 酶 活 性 } \\
\mathrm{cpm} / \mathrm{min} / \mathrm{ml} \text { 抆 }\end{array}$ & $\begin{array}{c}\text { 重组酶活性 } \\
\text { 回收率 }\end{array}$ \\
\hline 对 照 & 0 & 11306 & 100 \\
\hline 解离后 & 0.08 & 6325 & 1 \\
\hline 重组后 & 0.001 & 11257 & 99 \\
\hline
\end{tabular}

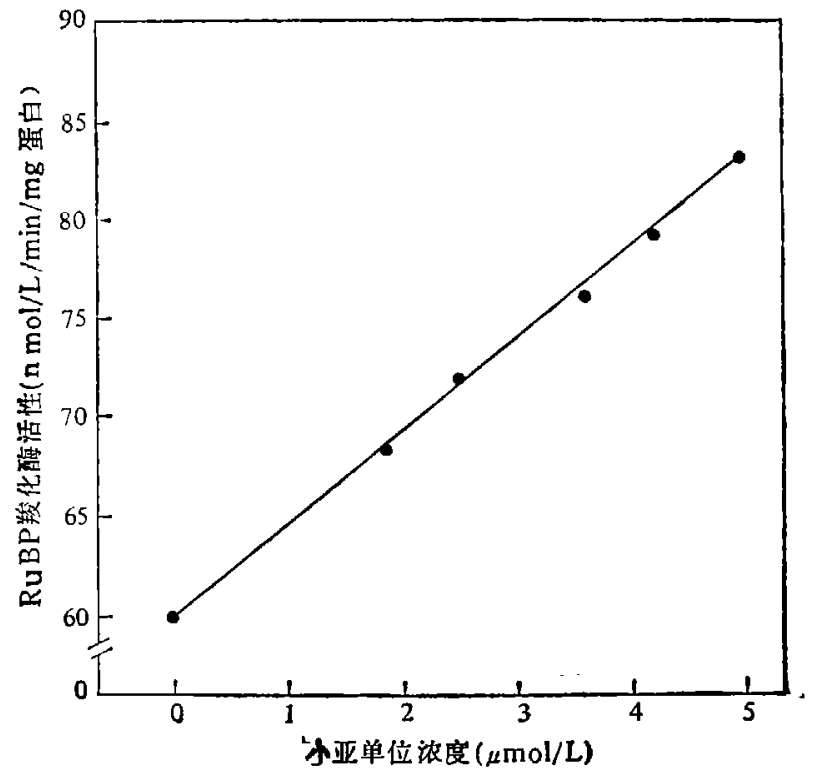

图 7 小亚单位浓度对缺陷小亚单位的固定化 RuBP 化酶活性的影响

\section{三、讨 论}

迄今从各种生物中来源的酶经固定化的至少有 200 种以上 ${ }^{[14]}$. RuBP 羧化酶的固定化则 尚未见有报道. 目前利用酶的固定化方法研究酶的结构与功能大多限于含同一种亚单位的室 聚酶,对于含不同亚单位的寡聚酶则研究较少. 由于高等植物 RuBP 羒化酶进行解离后, 当 除去解离剂时,极易发生不可逆的聚合沉淀,而 Nishimura 和 Akazawa ${ }^{[8]}$ 用碱的方法会促使 大亚单位核的完整性破坏,并促进亚单位之间的聚合作用 ${ }^{[6]}$. 由于高等植物 RuBP 羧化酶解 离及重组实验的困难，这一问题近年来并未取得进展。本文在非活化条件下，将烟草 RuBP 
羧化酶共价偶联于 CNBr 活化的 Sepharose 4B 载体上, 并以此为一种手段研究了原素对酶 的解离和重组作用,发现在低浓度尿素处理后可将酶的小亚单位和大亚单位核, $L_{\mathbf{g}}$ 分离. 由于 载体的位阳效应，当除去尿素后亚单位之间便不产生这种聚合沉淀作用，因此为研究高等植物 RuBP 羧化酶的解离和重组提供了新的手段. 本文结果表明：酶的活性的损失与小亚单位解 离的程度呈线性关系. 将尿素稀释,则解离的亚单位又可重组合,其酶活性的恢复亦呈线性关 系, 这说明酶的活化及催化部位虽然均在大亚单位上, 但小亚单位对酶的催化活性也起重要 影响.一般推测, 认为小亚单位可能为维持酶活性所需的一定构象中起作用 ${ }^{[15]}$. $\mathrm{Li}$ 等 ${ }^{[16]}$ 曾利 用种间杂交的方法将 RuBP 羒化酶的大、小亚单位进行了种间的重组,发现改变种间的小亚单 位对酶的动力学性质基本上没有影响，这可能是烟草种间小亚单位的差异尚不足以引起对酶 摧化和活化部位的构象引起明显的改变. 小亚单位在维持酶活性方面的作用机制将有待再进 一步研究.

Eisenberg 等 ${ }^{[17]}$ 根据 X-射线衍射图, 曾对 RuBP 羧化酶的四级结构提出了一个假设性 的模型, 认为 8 个大亚单位和 8 个小亚单位呈对称性排列为二层, 每一层为四个大亚单位和 四个小亚单位. 小亚单位的精确位置尚不清楚, 可能排列在大亚单位的周围. 我们发现 2.0 $\mathrm{mol} / \mathrm{L}$ 尿素可使小亚单位解离下来，而大亚单位仍偶联在载体上,这可以推测, 小亚单位可能 排列在大亚单位中间. 因此，当天然酶与载体混和时只有部份大亚单位才可直接与载体接触 起共价偶联反应。当低浓度尿素处理时，大、小亚单位之间相结合的键断裂，因此仅小亚单位 被解离下来。当尿素浓度达 $3.0 \mathrm{~mol} / \mathrm{L}$ 时,大亚单位核解离为单体.

现在, 我们对 Eisenberg 等 ${ }^{[17]}$ 提出的 RuBP 羧化酶四级结构模型又提出了一个新的证据.

本文承殷宏章、沈允钢、施教耐三位教授审阅，并提出宝贵意见，在此表示感谢。

\section{参考文献}

[1] Hartman, F. C. et al., in Photosynthesis IV Regulation of carbon metabolism (Ed. Akoyounoglou, G.), Philadelphia, Balaban Int. Sci. Serv, 1981, 17-29.

[2] I.orimer, C. H. \& Mizirko, H. M., Biochemistry, 19(1980), 5321-5328.

[3] Andrews, T. J. \& Ballment, B., J. Biol. Chem., 258(1983), 7514-7518.

[ 4 ] Asami, S. et a1., Arch. Biochem. Biophys., 225(1983), 713-721.

[5] Takabe, T. et al., ibid., 229(1984) 202-211.

[ 6$]$ Andrews, T. \& Abel, K. M., J. Biol. Chem., 256(1981), 8445-8451.

[7] Jodan, D. B, \& Chollet, R., Arch. Biochem. Biophys., 236(1985), 487-496

[8] Nishimura, M. \& Akazawa, T., Biochem. Biophys. Res. Commun., 59(1974), 584-590.

[ 9 ] Akazawa T., in Encyclopedia of Plant Physiology, New Series (Eds. Gibbs, M. and Latzko, E.), Vol. 6, SpringerVerlag Berlin, 1979, 208-229.

[10] 李立人、王维光, 植物生理学报, 11(1985), 110-112.

[11] Chan, WWC., in Methods in Enzymology (Ed. Mosbach, K.), Academic Press, Vol. VLIV 1976491.

[12] 李立人、王维光, 植物生理学报, 10(1984)，363-371.

[13] Weler, K. \& Osborn, M., J. Biol. Chem, 244(1969), 4406-4412.

[14] Agirre, R. H., Ymmobilization as a tool to investigate the function and stability of oligomeric enzymes, Minerva Publikation Munchen, 1982, 10-20.

[15] Mizirko, H. M. \& Lorimer, G. H., Ann. Rev. Biochem, 52(1983), 507-535.

[16] Li, L. R. et al., Plant Physiol, 71(1983), 404-408.

[17] Eisenberg. D. et al., in Photosynthetic Carbon Assimilation (Eds. Siegelman, H. W. \& Hind, G.), New York, Plenum, 1978, 271-281. 